\title{
Highlights of top-quark properties measurements at the ATLAS experiment *
}

\author{
O. Majerský, on behalf of the ATLAS Collaboration ${ }^{\mathrm{a}, \mathrm{b}}$ \\ ${ }^{a}$ Faculty of Mathematics, Physics and Informatics, Comenius University, Mlynská dolina 84248 Bratislava, Slovakia \\ ${ }^{b}$ Department of Nuclear and Particle Physics, University of Geneva, 24 quai Ernest-Ansermet, CH-1211 Geneva, Switzerland
}

\begin{abstract}
The top quark is the heaviest known fundamental particle. As it is the only quark that decays before it hadronizes, this gives a unique opportunity to probe the properties of pseudo-bare quarks. This proceeding focuses on a few recent precision measurements of top-quark properties in top-quark pair events by the ATLAS Collaboration using LHC proton-proton collision data. Among these is the measurement of color flow and measurement of spin correlations in $t \bar{t}$ pair production. Measurements of the top-quark mass and searches for rare top-quark decays are also presented.
\end{abstract}

Keywords: ATLAS experiment, spin correlations, top-quark mass, color flow, FCNC

\section{Introduction}

The top quark is the heaviest known elementary particle with the mass of around $173 \mathrm{GeV}$. According to the Standard Model (SM), the top quark is an unstable particle with extremely short life-time $\left(0.5 \times 10^{24} \mathrm{~s}\right)$ and thus it decays before it can hadronize. In the SM, the top quark decays almost exclusively into a $W b$ pair which inherits its properties unaffected by hadronization effects. It is therefore possible to precisely measure various properties of the top quark, such as the top-quark mass, spin correlations in top-quark pair production or the properties of the $W_{t b}$ vertex. Additionally, there are many Beyond-Standard-Model (BSM) theories that predict different values of some of the top-quark-related parameters. Therefore, measurements of the top-quark properties can also be a probe for the BSM physics.

At the Large Hadron Collider (LHC) [1], the top quark is produced abundantly via pair-production, allowing for precise studies of its properties. We present several recent ATLAS [2] measurements of top-quark

${ }^{*}$ Talk given at 22nd International Conference in Quantum Chromodynamics (QCD 19, 34th anniversary), 2 July - 5 July 2019, Montpellier - France

Email address: oliver.majersky@cern.ch (O. Majerský, on behalf of the ATLAS Collaboration) properties and searches for flavor-changing neutral currents in rare decays of top quarks heavily suppressed in SM.

\section{Measurement of spin correlations in the $t \bar{t}$ pair production}

At the LHC, top-quark pairs are produced unpolarized; however, the spin of the top quark and the top anti-quark are correlated. Since the top quark decays before hadronization, it is possible to measure observables sensitive to the spin correlations by reconstructing the decay products carrying the spin information of the top quarks. In particular, due to the $V-A$ structure of the decay of $W$ bosons, where $V$ is the vector current and $A$ is the axial current, the leptons from semi-leptonic decays of the top quarks are sensitive to the spin correlations [3,4]. ATLAS has measured observables sensitive to spin correlations in the dilepton channel, with a single isolated electron and a single isolated muon in the final state [5]. The measurement uses proton-proton $(p p)$ collision data collected by the ATLAS detector, corresponding to the integrated luminosity of $36.1 \mathrm{fb}^{-1}$ at the center-of-mass energy $\sqrt{s}=13 \mathrm{TeV}$. Unfolded differential cross sections are measured as a function of the 
azimuthal opening angle, $\Delta \phi_{\ell^{+} \ell^{-}}{ }^{1}$, and the pseudorapidity difference between the electron and muon candidate in the laboratory frame, $\left|\Delta \eta_{\ell^{+} \ell^{-}}\right|$. The measurement is performed inclusively as well as differentially as a function of the invariant mass of the reconstructed $t \bar{t}$ system, $m_{t \bar{t}}$.

In the inclusive measurement, at least two jets with $p_{\mathrm{T}}>25 \mathrm{GeV}$ are required, where at least one of the jets is required to be identified as initiated by a $b$-quark ( $b$-tagged). For the differential measurements, a tighter selection is imposed, where at least two jets are required to be $b$-tagged. A reconstruction of the full $t \bar{t}$ system is performed using the Neutrino Weighting method [6]. This methods attempts to determine the four-momenta of the two final-state neutrinos from the semi-leptonic $t \bar{t}$ decays using constraints on the $W$ mass, the top-quark mass and the measured missing transverse energy in the detector.

The measured observables are corrected for detector resolution and acceptance effects using an iterative Bayesian unfolding (IBU) method [7]. The observables are unfolded using two different definitions of particles in the Monte Carlo (MC) simulation: parton level and particle level. Parton-level objects are taken from the MC simulation, where the top quarks are considered after additional radiation but before decay. Parton-level leptons are taken before additional radiation. The measurement unfolded to parton level is extrapolated to full phase-space with all of the dilepton events considered, with the exception of the electrons and muons originating from intermediate $\tau$ decays from the $t \rightarrow W b \rightarrow \tau v b$ decay chain. The parton-level unfolded results can be directly compared to fixed-order theoretical predictions.

The particle-level objects are reconstructed from MC simulated events using only particles with lifetimes $t>$ $3 \times 10^{-11} \mathrm{~s}$, employing procedures similar to those used for detector-level object reconstruction for maximum correspondence. The measurement unfolded to particlelevel uses similar fiducial selection as the detector-level selection, where a jet is $b$-tagged if a $B$-hadron is associated to the jet using information from the MC simulation. In contrast to parton level, particle-level unfolded measurements are less sensitive to MC signal modelling uncertainties due to the use of more well-defined objects but cannot be directly used for comparison with fixedorder predictions.

The results of the measurement show a significant tension between the unfolded differential cross section

\footnotetext{
${ }^{1}$ The azimuthal opening angle $\Delta \phi_{\ell^{+} \ell^{-}}$is defined as the angle between the lepton candidates in the plane perpendicular to the beam line.
}

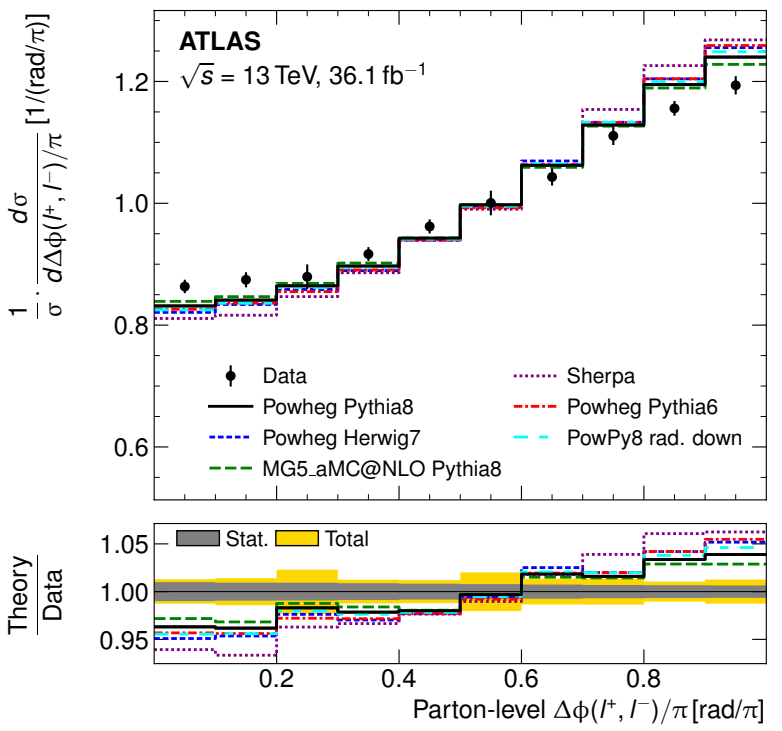

Figure 1: Comparison of differential cross section as a function of the parton-level $\Delta \phi_{\ell^{+} \ell^{-}}$with prediction obtained using various MC generators [5].

as a function of $\Delta \phi_{\ell^{+} \ell^{-}}$and the MC predictions, shown in Figure 1. The compatibility of the observed level of spin correlations with the SM prediction is quantified using a template fit to the unfolded parton-level $\Delta \phi_{\ell^{+} \ell^{-}}$differential cross section. Two templates are constructed; one using the nominal Powheg+Pythia8 MC prediction with the "SM-like spin correlation" and an additional template using PowHEG+Pythia8 interfaced with MADSpIN with spin correlations disabled. The obtained fraction of SM-like spin correlation $f_{\mathrm{SM}}=$ $1.249 \pm 0.024$ (stat.) \pm 0.061 (syst.) \pm 0.040 (theor.) deviates from the nominal $\mathrm{MC}$ prediction with a significance of $3.2 \sigma$, where the MC prediction is based on NLO QCD calculation. The limiting factor of the measurement of $f_{\mathrm{SM}}$ are the uncertainties on the $t \bar{t}$ modelling.

Several alternative improved predictions are compared with data, as shown in Figure 2. These include MC prediction obtained using MCFM generator, which simulates the full NLO top-quark decay including offshell effects, both of which are absent in the nominal Powheg+Pythia8 prediction which uses the narrowwidth approximation. The MCFM prediction does not show any reduction in the observed discrepancy. Additionally, the nominal prediction is reweighted using NNLO QCD calculation, slightly reducing the tension with the unfolded data. Finally, an NLO QCD + NLO electroweak prediction with a fixed choice of renormal- 


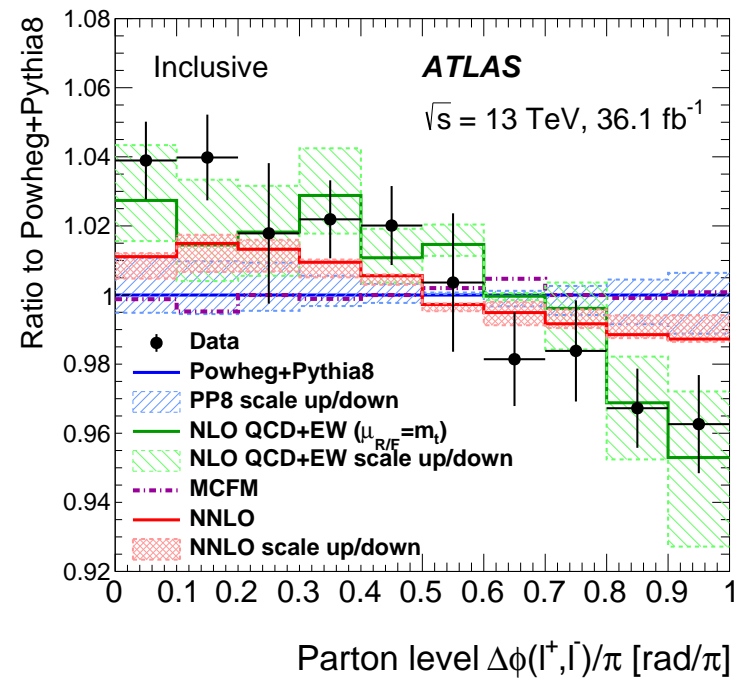

Figure 2: Comparison of differential cross section as a function of the parton-level $\Delta \phi_{\ell^{+} \ell^{-}}$with additional theory predictions [5]. The ratio in the plot is centered on the nominal MC prediction simulated by POWHEG+PyThia8.

ization and factorization scale is found to be compatible with the unfolded data, although this prediction has a large theoretical uncertainty arising from the variation of the scales.

\section{Measurement of color-flow observables in $t \bar{t}$ pair production}

The color flow between strongly-interacting particles is one of the least experimentally validated effects of QCD theory, typically described by a phenomenological approach due to the non-perturbative regime of these interactions. ATLAS has measured jet-constituent observables sensitive to color flow in $t \bar{t}$ events using $36 \mathrm{fb}^{-1}$ of $p p$ collision data at $\sqrt{s}=13 \mathrm{TeV}$ [8]. The observable of interest in this measurement is the jet-pull vector, which is a transverse-momentum-weighted radial moment of the jet:

$$
\overrightarrow{\mathcal{P}}(j)=\sum_{i \in j} \frac{\left|\overrightarrow{\Delta r_{i}}\right| \cdot p_{\mathrm{T}}^{i}}{p_{\mathrm{T}}^{j}} \overrightarrow{\Delta r_{i}},
$$

where $p_{\mathrm{T}}^{i}$ is the transverse momentum of the $i$-th constituent of jet $j$ and $\overrightarrow{\Delta r_{i}}=\left(\Delta y_{i}, \Delta \phi_{i}\right)$ is the distance of the constituent from the reconstructed jet axis in the space of rapidity and azimuthal angle $(y-\phi)$.

For a system of two jets $j_{1}$ and $j_{2}$, a jet-pull angle $\theta_{\mathcal{P}}\left(j_{1}, j_{2}\right)$ can be defined as the angle between the jet- pull vector $\overrightarrow{\mathcal{P}}\left(j_{1}\right)$ and the vector connecting $j_{1}$ to $j_{2}$ in the $(y-\phi)$ space, defined as $\left(y_{j_{2}}-y_{j_{1}}, \phi_{j_{2}}-\phi_{j_{1}}\right)$. For a pair of jets which are color-connected, QCD predicts an increase of radiation affecting the structure of the jet [9], due to which the jet-pull vector and the jet connection vector are expected to be aligned. Thus the jet-pull angle is expected to be centered around zero. In contrast, for jets which are not color-connected, the pull angle is expected to be distributed uniformly between $-\pi$ and $\pi$. The measurement is performed using $t \bar{t}$ events with an isolated electron or muon and four jets in final state. The four jets naturally form two pairs with respect to color flow. The jets originating from the hadronic decay of $W$ boson are color-connected due to the initiating quarks originating from the colorless $W$ boson. The jets originating from $b$ quarks from the $t$ and $\bar{t}$ decay are not color-connected. The measured observables are thus the jet-pull angle and the magnitude of the jet-pull vector for the light-quark ${ }^{2}$ jet and $b$-jet pairs. The observables are calculated from tracks associated to the jets due to the superior spatial resolution of the inner detector when compared to the granularity of the ATLAS calorimeter system [2].

The measured observables are corrected for detector resolution and acceptance effects using the IBU method, following a particle-level definition of objects very similar to the spin correlations measurement in Sec. 2.

A comparison of the unfolded normalized differential cross sections with various $t \bar{t}$ MC generators for a subset of the observables of interest is shown in Figure 3 and 4. The uncertainties on these unfolded data to MC comparisons are dominated by $t \bar{t}$ modelling, $b$-tagging and background modelling-related uncertainties. In the comparisons, the most relevant difference between the various generator predictions is the choice of the generator responsible for showering and hadronization modelling. While Pythia uses the color string model [10], Herwig uses the cluster model [11] for hadronization. Finally, SHERPa uses a different implementation of the cluster model compared to Herwig. For each of the observables, a suitable choice of MC generator prediction may describe the unfolded data well, but none of the tested MC generators can simultaneously describe all of the measured observables.

\section{Direct measurement of the top-quark mass}

The mass of the top quark $\left(m_{\mathrm{top}}\right)$ is one of the fundamental parameters of the SM. It plays an important

\footnotetext{
${ }^{2}$ We refer to jets initiated by gluon and or quark excluding bottom and top quark.
} 


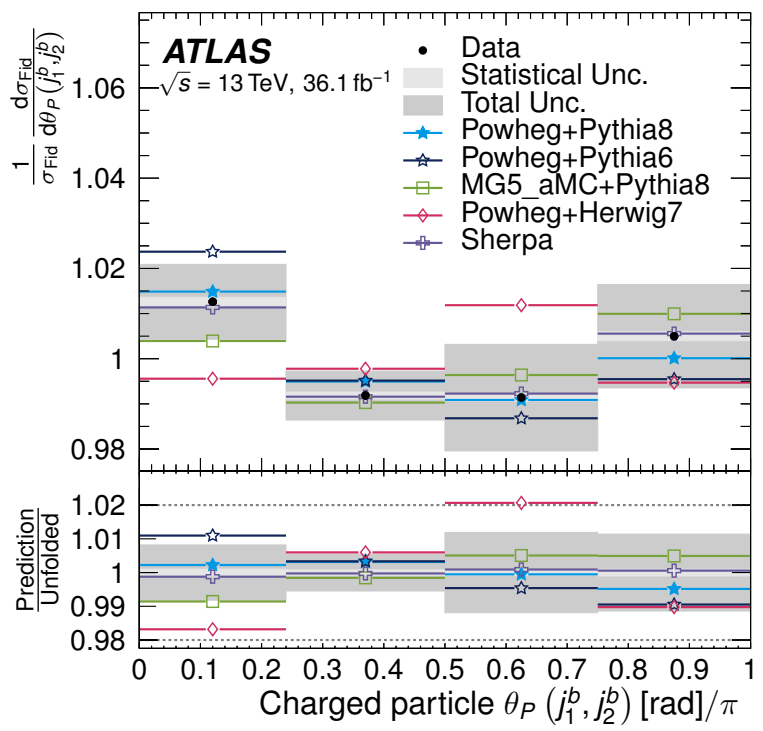

Figure 3: Comparison of unfolded normalized differential cross section as a function of $b$-jet pair jet-pull angle with MC prediction of various MC generator setups used in ATLAS [8].

role in radiative corrections of electroweak processes. These include loop corrections which bind together the $m_{\text {top }}$ with the mass of the Higgs boson and the $W$ boson mass. A precise determination of $m_{\text {top }}$ is necessary for a test of internal consistency of the SM as well as for the determination of the stability of the electroweak vacuum.

The measurement presented here is performed using $20.2 \mathrm{fb}^{-1}$ of $p p$ collision data at $\sqrt{s}=8 \mathrm{TeV}$ in $t \bar{t}$ events with a single isolated lepton in final state [12]. The $m_{\text {top }}$ is extracted using an elaborate template fit technique. A three-dimensional template fit is performed using templates generated from MC simulation with different values of three underlying parameters. The varied parameters are the value of $m_{\text {top }}$ in $\mathrm{MC}^{3}$, the jet energy scale and the ratio of $b$-to-light-quark jet energy scale. The corresponding fit template distributions sensitive to the respective fit parameters are the reconstructed top-quark mass distribution $m_{\mathrm{top}}^{\text {reco }}$ (Figure 5), the reconstructed $W$ boson mass and the ratio of $b$-jet to light-quark jet sum of $p_{\mathrm{T}}$. The latter two parameters are included in the fit to reduce the impact of the jet and $b$-jet energy scale uncertainties on the measured $m_{\text {top }}$. The events selected for the measurement are required to include an isolated electron or muon and at least four jets, out of which at least two jets are $b$-tagged. A boosted decision tree

\footnotetext{
${ }^{3}$ The $m_{\text {top }}$ parameter in MC is commonly treated as the pole mass despite the known theoretical ambiguity in its definition.
}

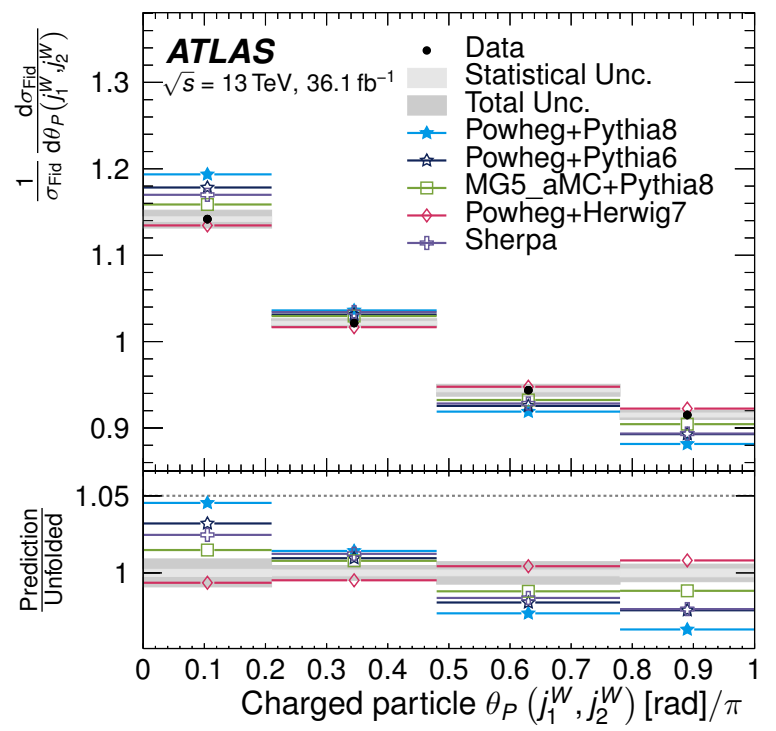

Figure 4: Comparison of unfolded normalized differential cross section as a function of color-connected light-quark jet-pull angle with MC prediction of various MC generator setups used in ATLAS [8].

(BDT) multivariate technique is trained using 13 variables related to the event kinematics to determine correct jet-to-parton assignment in the event. A selection on the trained BDT classifier is imposed to reject background events as well as $t \bar{t}$ events with incorrectly assigned jets. An optimization of the BDT cut is performed with the aim to reduce the total uncertainty on the measurement, in particular leading to a dramatic reduction in the size of $t \bar{t}$ modelling uncertainties.

The measured result is $m_{\text {top }}=172.08 \pm 0.39$ (stat.) \pm 0.82 (syst.) GeV. The measurement is dominated by systematic uncertainties on jet energy scale and $b$-tagging, followed by $t \bar{t}$ modelling uncertainties. The combination with previous ATLAS measurements gives $m_{\text {top }}=$ $172.69 \pm 0.25$ (stat.) \pm 0.41 (syst.) GeV.

\section{Measurement of top-quark mass in $t \bar{t}$ events with additional jet}

The direct approach to $m_{\text {top }}$ extraction presented in Sec. 4 is currently the approach with the smallest experimental uncertainties. However, the relation between the $m_{\text {top }}$ extracted in direct measurements and the topquark pole mass contains an ambiguity due to nonperturbative effects in the MC simulation, resulting in a theoretical uncertainty of $\approx 0.5 \mathrm{GeV}$.

A different approach to extraction of the top-quark pole mass $m_{\text {top }}^{\text {pole }}$ is performed in the ATLAS measurement of the top-quark mass in $t \bar{t}$ events with isolated 


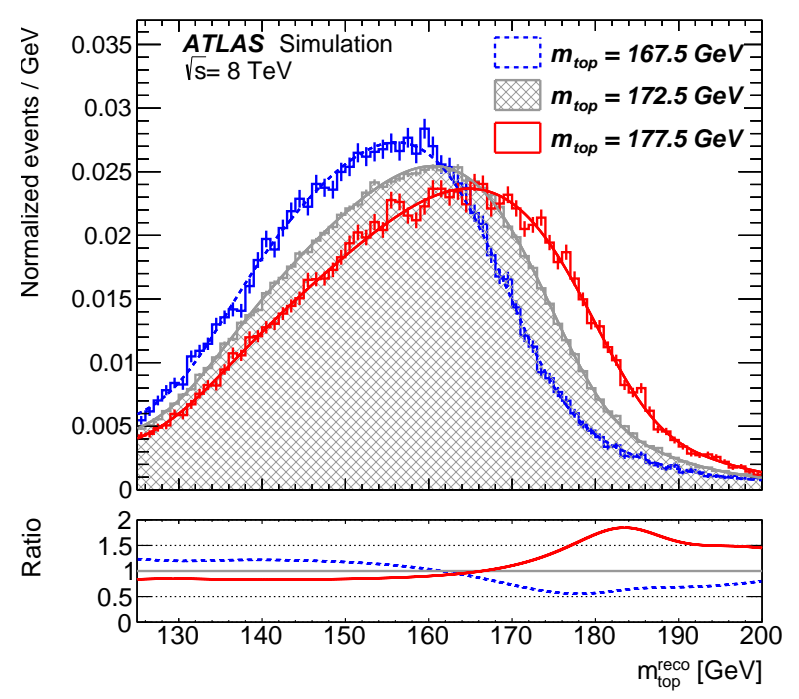

Figure 5: Distribution of the $m_{\text {top }}^{\text {reco }}$ for MC simulation with various values of the MC $m_{\text {top }}$ parameter [12].

lepton and an extra jet in final state using $20.2 \mathrm{fb}^{-1}$ of $p p$ collision data at $\sqrt{s}=8 \mathrm{TeV}$ [13]. The measured observable is an unfolded normalized differential cross section as a function of observable sensitive to the $m_{\mathrm{top}}$, which is then compared to a fixed-order theory prediction that uses a well-defined renormalization scheme to extract the $m_{\text {top }}$.

The measurement uses events with a single isolated electron or muon and at least five jets, out of which two are $b$-tagged. A set of kinematic criteria is used to reconstruct the $t \bar{t}+1 j$ system. The observable considered in the measurement sensitive to the top-quark mass is the $\rho_{s}$ :

$$
\rho_{s}=\frac{2 m_{0}}{m_{t \bar{t}+1 j}}, \quad m_{0}=170 \mathrm{GeV} .
$$

The value of $m_{0}$ in $\rho_{s}$ is arbitrary, but conveniently chosen to be approximately the top-quark mass. The $m_{t \bar{t}+1 j}$ is the mass of the reconstructed $t \bar{t}$ system with the additional jet. An important feature of this observable is that it is not sensitive to incorrect assignment of jets to partons in the reconstruction.

The measured normalized differential cross section as a function of $\rho_{s}$ is corrected for detector acceptance and resolution effects using the IBU method to both partonlevel and particle-level. The parton-level differential cross section is compared to a fixed-order NLO QCD prediction and the value of the top-quark mass is extracted using a $\chi^{2}$ fit in both pole-mass and $\overline{\mathrm{MS}}$ renormalization schemes. A comparison of the parton-level differential cross section with the NLO QCD prediction

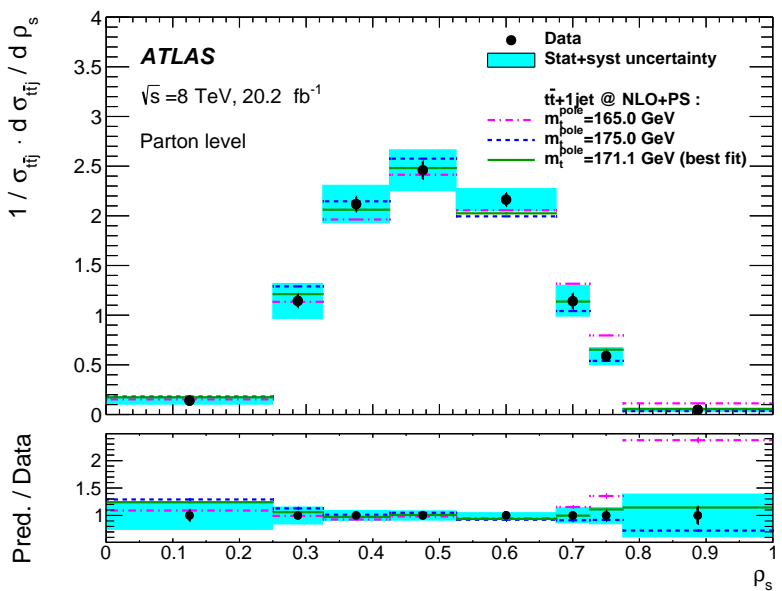

Figure 6: The unfolded parton-level normalized differential cross section as a function of the $\rho_{s}$ observable, compared to fixed-order NLO QCD prediction for different values of the top-mass (colored lines). The best-fit prediction is shown in a green solid line. [13]

for a few values of the $m_{\text {top }}^{\text {pole }}$ and the best-fit prediction is shown in Figure 6. The measured values of the topquark mass in the pole-mass and $\overline{\mathrm{MS}}$ scheme, respectively, are:

$$
\begin{aligned}
& \left.m_{\text {top }}^{\text {pole }}=171.1 \pm 0.4(\text { stat. }) \pm 0.9(\text { syst. })_{-0.3}^{+0.7} \text { (theo. }\right) \mathrm{GeV} \\
& \left.m_{\text {top }}^{\overline{\mathrm{MS}}}=162.9 \pm 0.5(\text { stat. }) \pm 1.0(\text { syst. })_{-1.2}^{+2.1} \text { (theo. }\right) \mathrm{GeV}
\end{aligned}
$$

The measurement is limited by systematic uncertainties on $t \bar{t}$ modelling of showering, hadronization and color reconnection as well as uncertainty on the jet energy scale.

A comparison of the previous $m_{\text {top }}^{\text {pole }}$ measurements with the current best $m_{\text {top }}^{\text {pole }}$ measurement as well as direct $m_{\text {top }}$ measurement is shown in Figure 7 . The $8 \mathrm{TeV}$ $m_{\text {top }}^{\text {pole }}$ measurement presented here shows a reduction in uncertainty of a factor of 2 , reaching a precision close to $1 \mathrm{GeV}$. It can be therefore expected that future $m_{\mathrm{top}}^{\text {pole }}$ measurements may further challenge the theoretical interpretations of the top-quark mass.

\section{Search for flavor-changing neutral currents in rare top decays}

The SM prohibits flavor-changing neutral currents (FCNC) in decays at leading order, and higher-order corrections are heavily suppressed via the GIM mechanism [15]. Evidence of an otherwise-forbidden decay would therefore be a sign of possible BSM physics. ATLAS has performed searches for FCNC in top-quark 


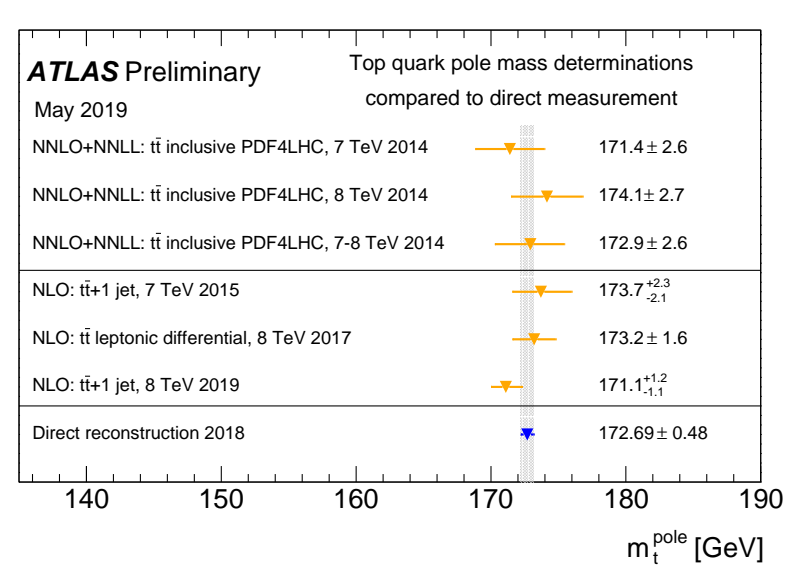

Figure 7: Comparison of ATLAS $m_{\text {top }}^{\text {pole }}$ measurements to the direct $m_{\text {top }}$ measurement [14].

decays. Here we present a search for $t \rightarrow H q(q=u, c)$ decay in $t \bar{t}$ events [16]. The search is performed using $36.1 \mathrm{fb}^{-1}$ of $p p$ collision data at $\sqrt{s}=13 \mathrm{TeV}$. Two different decay channels are considered based on the Higgs boson decay: $t \rightarrow H(b \bar{b}) q$ and $t \rightarrow H\left(\tau^{+} \tau^{-}\right) q$.

\section{1. $t \rightarrow H(b \bar{b}) q$ channel}

The search for FCNC in $t \rightarrow H(b \bar{b}) q$ decays is performed in events with a single isolated lepton, assumed to originate from the SM-like semi-leptonic decay of the other top quark, and 4 jets, out of which 3 must be $b$-tagged. Regions with additional jets from initialand final-state radiation are also considered to increase sensitivity. Fig 8 shows the expected yields for backgrounds and signal prediction for a branching fraction $\mathcal{B}=1 \%$. A likelihood discriminant is constructed for the signal hypothesis $(t \bar{t} \rightarrow W b H q)$ and for the background hypothesis $(t \bar{t} \rightarrow W b W b)$. A comparison of data with prediction for the likelihood discriminant in one of the most sensitive regions is shown in Figure 9. The likelihood discriminant is simultaneously fit in multiple regions to data using a binned profile likelihood fit including systematic uncertainties as additional nuisance parameters. The parameter of interest is the signal strength with respect to the $\mathcal{B}=1 \%$ assumption. The best-fit value of $\mathcal{B}(t \rightarrow H c)$ is obtained assuming $\mathcal{B}(t \rightarrow H u)=0$ and vice-versa. The search is limited by systematic uncertainties, among which the dominant sources are the modelling of the associated production of $t \bar{t}$ and additional jets background and the $b$-tagging-related systematic uncertainties. No significant excess compared to the SM-only background hypothesis is found, and the observed (expected) limits at

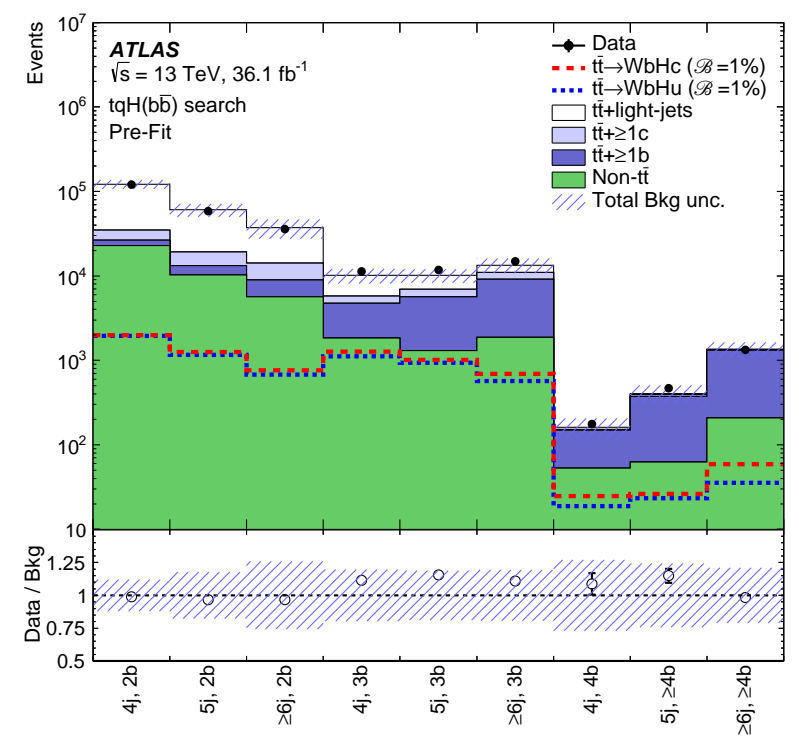

Figure 8: A comparison of predicted yields with data for the regions considered in the $t \rightarrow q H(b \bar{b})$ search [16]. A signal prediction (dashed blue and red line) is shown for the assumption of branching ratio $\mathcal{B}(t \rightarrow H(b \bar{b}) q)=1 \%$.

the $95 \%$ confidence level (CL) are calculated for the branching ratios:

$$
\begin{aligned}
& \mathcal{B}(t \rightarrow H c)=4.2 \times 10^{-3}\left(4.0 \times 10^{-3}\right), \\
& \mathcal{B}(t \rightarrow H u)=5.2 \times 10^{-3}\left(4.9 \times 10^{-3}\right)
\end{aligned}
$$

\section{2. $t \rightarrow H\left(\tau^{+} \tau^{-}\right) q$ channel}

In the case of the $t \rightarrow H\left(\tau^{+} \tau^{-}\right) q$ channel, the FCNC search is performed under two event signature assumptions, either both $\tau$ leptons decaying hadronically, or a single hadronic $\tau$ decay and a single leptonic $\tau$ decay. For each of the $\tau$ decay scenarios, a region with exactly 3 jets and a region with $\geq 4$ jets is considered. In each region a BDT is trained to separate signal and background using multiple kinematic-related variables.

In a manner similar to the $t \rightarrow H(b \bar{b}) q$ search, the BDT discriminant is simultaneously fit in multiple regions using binned profile likelihood fit including systematic uncertainties as additional nuisance parameters. The sensitivity of the search is limited by statistical uncertainty as well as some of the systematic uncertainties, in particular the normalisation uncertainty of the datadriven fake $\tau$ background estimate and the jet energy scale uncertainties. No significant excess compared to the SM-only background hypothesis is found and the 
observed (expected) limits at the $95 \%$ CL are calculated for the branching ratios:

$$
\begin{aligned}
& \mathcal{B}(t \rightarrow H c)=1.9 \times 10^{-3}\left(2.1 \times 10^{-3}\right), \\
& \mathcal{B}(t \rightarrow H u)=1.7 \times 10^{-3}\left(2.0 \times 10^{-3}\right) .
\end{aligned}
$$

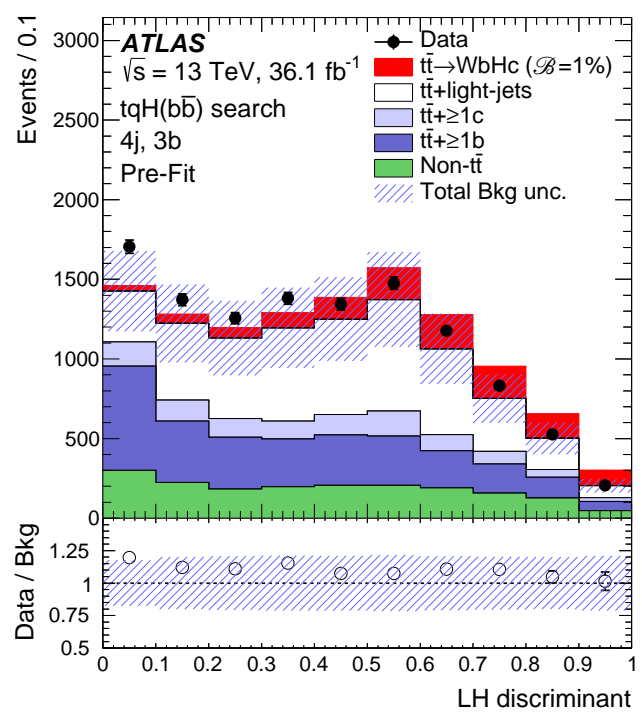

Figure 9: Comparison of data and prediction for the likelihood discriminant in the 4-jet region with highest signal sensitivity [16]. The red histogram shows the signal contribution prediction assuming a branching ratio $\mathcal{B}(t \rightarrow H(b \bar{b}) c)=1 \%$.

\subsection{Combination with previous ATLAS $13 \mathrm{TeV} t \rightarrow H q$ FCNC searches}

The $t \rightarrow H(b \bar{b}) q$ and $t \rightarrow H\left(\tau^{+} \tau^{-}\right) q$ channel results are combined with the previous ATLAS $13 \mathrm{TeV}$ $t \rightarrow H q$ measurements considering other Higgs-boson decay channels: $H \rightarrow \gamma \gamma$ [17] and $H \rightarrow W W, Z Z, \tau \tau$ with multiple leptons in final state [18]. A comparison of the upper limits on the branching ratio of $\mathcal{B}(t \rightarrow H c)$ and $\mathcal{B}(t \rightarrow H u)$ is shown in Figures 10 and 11, respectively. The combined observed (expected) limits at the $95 \%$ CL for the branching ratios are:

$$
\begin{aligned}
& \mathcal{B}(t \rightarrow H c)=1.2 \times 10^{-3}\left(0.83 \times 10^{-3}\right), \\
& \mathcal{B}(t \rightarrow H u)=1.1 \times 10^{-3}\left(0.83 \times 10^{-3}\right) .
\end{aligned}
$$

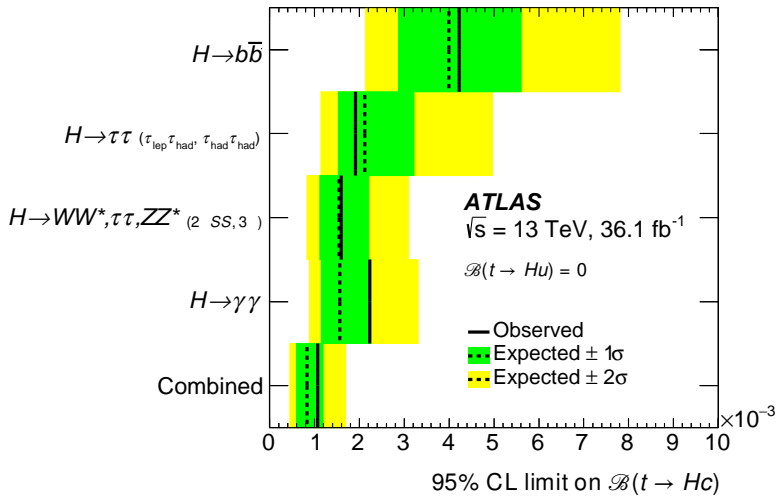

Figure 10: Comparison of the expected and observed limits on the $\mathcal{B}(t \rightarrow H c)$ in the individual ATLAS FCNC $13 \mathrm{TeV}$ measurements and the combination of these measurements [16], assuming $\mathcal{B}(t \rightarrow$ $H u)=0$.

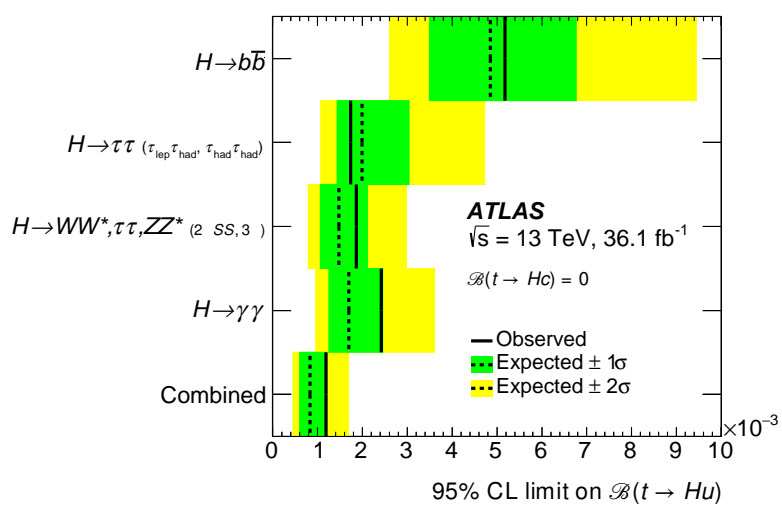

Figure 11: Comparison of the expected and observed limits on the $\mathcal{B}(t \rightarrow H u)$ in the individual ATLAS FCNC $13 \mathrm{TeV}$ measurements and the combination of these measurements [16], assuming $\mathcal{B}(t \rightarrow$ $H c)=0$.

\section{Conclusions}

In this proceeding we review the most recent ATLAS measurements of some of the top-quark properties as well as searches for FCNC in top-quark decays, using LHC $p p$ collisions. The latest $13 \mathrm{TeV}$ measurement of the spin correlations in $t \bar{t}$ decays is reported, showing tension with the SM in the prediction by the commonly-used MC generator setup in ATLAS. Further theoretical progress is needed to understand the discrepancy. On a similar note related to the MC modelling, the latest $13 \mathrm{TeV}$ ATLAS measurement of the unfolded observables sensitive to color flow motivates further work on the phenomenological models to improve the hadronization modelling in MC generators. 
The latest ATLAS direct measurement of the topquark mass as well as the indirect measurement of the pole mass using $8 \mathrm{TeV}$ datasets show significant improvements in precision. New searches for FCNC in $t \rightarrow H q$ decays in $t \bar{t}$ events using partial $13 \mathrm{TeV}$ ATLAS dataset are also presented. No significant excess compared to SM background-only prediction is observed.

Copyright 2019 CERN for the benefit of the ATLAS Collaboration. Reproduction of this article or parts of it is allowed as specified in the CC-BY-4.0 license.

\section{References}

[1] L. Evans, P. Bryant, LHC Machine, JINST 3 (2008) S08001.

[2] ATLAS Collaboration, The ATLAS Experiment at the CERN Large Hadron Collider, JINST 3 (2008) S08003.

[3] M. Jeżabek, J. H. Kühn, Lepton spectra from heavy quark decay, Nucl. Phys. B 320 (1989) 20.

[4] S. J. Parke, Top Quark Spin Correlations - Theory, Nuovo Cim. C035N3 (2012) 111-114.

[5] ATLAS Collaboration, Measurements of top-quark pair spin correlations in the $e \mu$ channel at $\sqrt{s}=13 \mathrm{TeV}$ using $p p$ collisions in the ATLAS detector (2019). arXiv:hep-ex/1903.07570.

[6] D0 Collaboration, Measurement of the top quark mass using dilepton events, Phys. Rev. Lett. 80 (1998) 2063-2068.

[7] G. D'Agostini, A Multidimensional unfolding method based on Bayes' theorem, Nucl. Instrum. Meth. A 362 (1995) 487-498.

[8] ATLAS Collaboration, Measurement of colour flow using jetpull observables in $t \bar{t}$ events with the ATLAS experiment at $\sqrt{s}=13 \mathrm{TeV}$, Eur Phys. J. C 78 (10) (2018) 847.

[9] R. K. Ellis, W. J. Stirling, B. R. Webber, QCD and Collider Physics, Cambridge University Press, 2003.

[10] B. Andersson, G. Gustafson, G. Ingelman, T. Sjöstrand, Parton Fragmentation and String Dynamics, Phys. Rept. 97 (1983) 31145.

[11] J. Bellm, et al., Herwig 7.0/Herwig++ 3.0 release note, Eur. Phys. J. C 76 (4) (2016) 196.

[12] ATLAS Collaboration, Measurement of the top quark mass in the $t \bar{t} \rightarrow$ lepton + jets channel from $\sqrt{s}=8 \mathrm{TeV}$ ATLAS data and combination with previous results, Eur. Phys. J. C 79 (4) (2019) 290.

[13] ATLAS Collaboration, Measurement of the top-quark mass in $t \bar{t}+1$-jet events collected with the ATLAS detector in $p p$ collisions at $\sqrt{s}=8 \mathrm{TeV}$ (2019).

[14] ATLAS Collaboration, Top Quark Mass Summary Plots, ATLCOM-PHYS-2019-500, https://cds.cern.ch/record/2673730 (May 2019).

[15] L. Maiani, The GIM Mechanism: origin, predictions and recent uses, in: Proceedings, 48th Rencontres de Moriond on Electroweak Interactions and Unified Theories: La Thuile, Italy, March 2-9, 2013, pp. 3-16.

[16] ATLAS Collaboration, Search for top-quark decays $t \rightarrow H q$ with $36 \mathrm{fb}^{-1}$ of $p p$ collision data at $\sqrt{s}=13 \mathrm{TeV}$ with the ATLAS detector, JHEP 05 (2019) 123.

[17] ATLAS Collaboration, Search for top quark decays $t \rightarrow q H$, with $H \rightarrow \gamma \gamma$, in $\sqrt{s}=13 \mathrm{TeV} p p$ collisions using the ATLAS detector, JHEP 10 (2017) 129

[18] ATLAS Collaboration, Search for flavor-changing neutral currents in top quark decays $t \rightarrow H c$ and $t \rightarrow H u$ in multilepton final states in proton-proton collisions at $\sqrt{s}=13 \mathrm{TeV}$ with the ATLAS detector, Phys. Rev. D 98 (3) (2018) 032002. 